\title{
A Computational Investigation of the Photochemical Reaction Path of some Synthesized and Experimentally Analyzed Small-Chain Conjugated Nitrones
}

\author{
Praveen Saini ${ }^{\mathrm{a}}$, Mainak Banerjee ${ }^{\mathrm{a}}$, Anjan Chattopadhyay ${ }^{\mathrm{a} *}$ \\ ${ }^{a}$ Department of Chemistry, Birla Institute of Technology and Science (BITS), Pilani-K.K. Birla \\ Goa Campus, Goa, 403 726, India
}

\section{Supplementary Information}

General procedure for the synthesis of nitrones....................................2

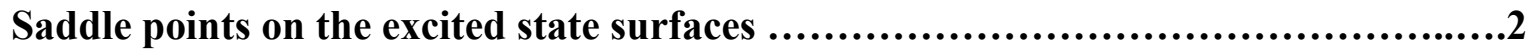

Optimized saddle points with structural parameters Figure $\mathrm{S} 1$........................

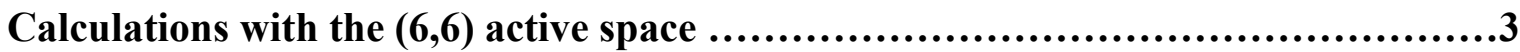

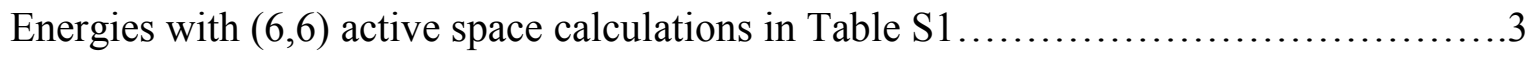

Conical intersection geometries at CASSCF $(6,6)$ level of calculations in Figure S2......4

Sloped Conical intersection of nitrone B $\ldots \ldots \ldots \ldots \ldots \ldots \ldots \ldots \ldots \ldots \ldots \ldots \ldots \ldots \ldots \ldots \ldots \ldots \ldots . . . . .5$

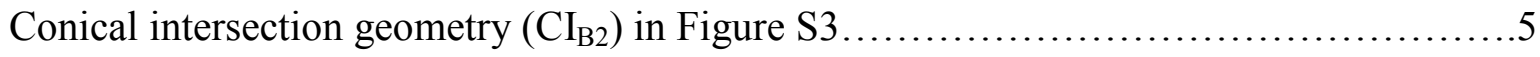

Cartesian coordinates of important geometries...................................5-18 


\section{General procedure for the synthesis of nitrones}

$N$-methyl hydroxylamine ( $1 \mathrm{mmol}$ ) was taken in a mortar (Agate made) and was gently ground by a pestle to make fine dust. To it respective aromatic aldehyde (Cinnamaldehyde or $\beta$-phenyl Cinnamaldehyde, $0.5 \mathrm{mmol}$ ) was added and the mixture was gently ground for another $2 \mathrm{~min}$. To this mixture sodium bicarbonate $(1.1 \mathrm{mmol})$ was added followed by addition of $200 \mu \mathrm{L}$ of $50 \%$ EtOH- $\mathrm{H}_{2} \mathrm{O}$ and the mixture was ground further for 15 minutes. The progress of the reaction was monitored by TLC. The crude reaction mixture was purified by column chromatography (silica gel, 60-120 mesh) using 5\% MeOH in Chloroform.

\section{Nitrone A: $(1 Z, 2 E)-N$-methyl-3-phenylprop-2-en-1-imine oxide}

${ }^{1} \mathrm{H}$ NMR (300 MHz, $\left.\mathrm{CDCl}_{3}\right): \delta(\mathrm{ppm}) 3.71(\mathrm{~s}, 3 \mathrm{H}), 6.93(\mathrm{~d}, J=16.2 \mathrm{~Hz}, 1 \mathrm{H}), 7.22(\mathrm{~d}, J=9.6$ $\mathrm{Hz}, 1 \mathrm{H}), 7.27-7.89(\mathrm{~m}, 6 \mathrm{H}) ;{ }^{13} \mathrm{C} \mathrm{NMR}\left(75 \mathrm{MHz}, \mathrm{CDCl}_{3}\right): \delta$ (ppm) 52.3, 118.4, 127.3, 128.8, 129.2, 136.0, 137.6, 138.1; IR (ATR): 1560, 1400, 1138, $974 \mathrm{~cm}^{-1}$; HRMS (ESI, $\left.\mathrm{m} / z\right)$ calcd for $\mathrm{C}_{10} \mathrm{H}_{11} \mathrm{NO}[\mathrm{M}+\mathrm{Na}]^{+}$184.0733, found: 184.0715 .

Nitrone B: (1Z)-N-methyl-3,3-diphenylprop-2-en-1-imine oxide

${ }^{1} \mathrm{H}$ NMR (400 MHz, $\left.\mathrm{CDCl}_{3}\right): \delta(\mathrm{ppm}) 3.66(\mathrm{~s}, 3 \mathrm{H}), 7.03(\mathrm{~d}, J=12.0 \mathrm{~Hz}, 1 \mathrm{H}), 7.23(\mathrm{~d}, J=8.0$ $\mathrm{Hz}, 1 \mathrm{H}), 7.29-7.45(\mathrm{~m}, 10 \mathrm{H}) ;{ }^{13} \mathrm{C} \mathrm{NMR}\left(100 \mathrm{MHz}, \mathrm{CDCl}_{3}\right): \delta(\mathrm{ppm}) 52.6,117.2,128.3,128.5$, 128.6, 128.9, 130.1, 136.6, 138.6, 140.5, 149.6; IR (ATR): 1541, 1404, $1122 \mathrm{~cm}^{-1}$; HRMS (ESI, $m / z)$ calcd for $\mathrm{C}_{16} \mathrm{H}_{15} \mathrm{NO}[\mathrm{M}+\mathrm{Na}]^{+} 260.1046$, found: 260.1047 .

\section{Saddle points on the excited state surfaces}

We have identified some important saddle points on the $S_{1}$ surfaces of both the nitrones (Figure $\mathrm{S} 1$ ). In nitrone $\mathbf{A}$, a transition state $\left(\mathbf{T S}_{\mathbf{A}}\right)$ is found to be situated at only $1 \mathrm{kcal} / \mathrm{mol}$ above the relaxed excited state geometry (and $7 \mathrm{kcal} /$ mol below the vertically excited geometry) with an imaginary frequency of $156 i \mathrm{~cm}^{-1}$. On the other hand, we have identified a saddle point (SP) on the first excited state surface of nitrone $\mathbf{B}$ which lies around 6-7 kcal/mol (Table 5) above the vertically excited geometry of this state at the CASSCF level. However, this geometry is found to be situated slightly below the Frank-Condon excited state at the CASPT2 level. It seems that this saddle point leads towards a transition state $\left(\mathbf{T S}_{\mathbf{B} 1}\right)$ with an imaginary frequency of $51 i \mathrm{~cm}^{-1}$ showing twist in the CNO region (quite similar to the twist shown by $31 i \mathrm{~cm}^{-1}$ frequency of $\mathbf{S P}$ ). Our results have indicated that these geometries play a key role during the passage towards the oxaziridine formation. Interestingly, this important saddle point found on the first excited state surface of nitrone $\mathbf{B}$ has been identified only at the $(4,4)$ level of calculations. Another transition state $\left(\mathbf{T S}_{\mathbf{B} 2}\right)$ in nitrone $\mathbf{B}$ has been detected on the first excited state surface which is found to create a very high barrier and is situated around $25 \mathrm{kcal} / \mathrm{mol}$ above the vertically excited point at the CASSCF level. 


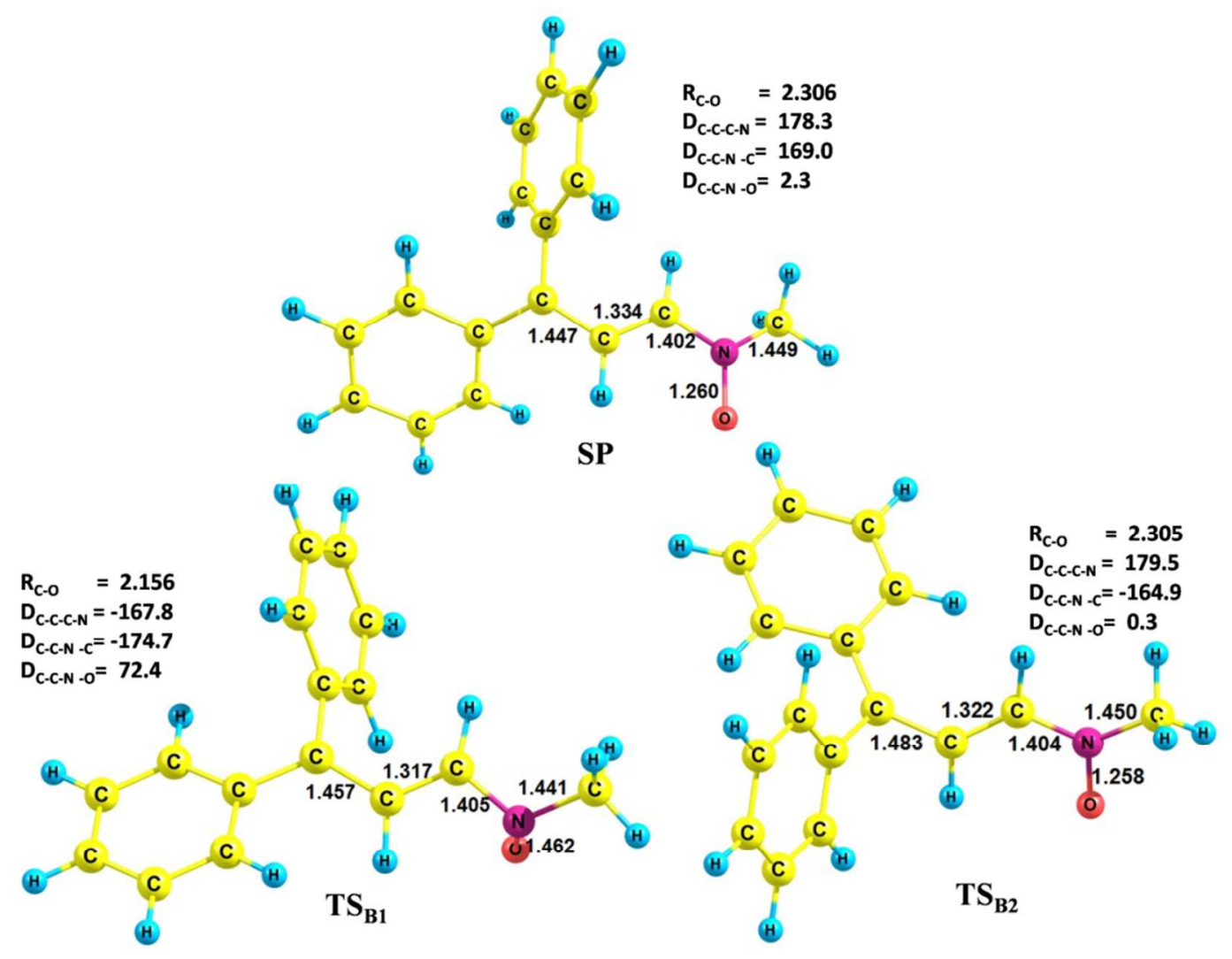

Figure S1: Optimized saddle point $(\mathbf{S P})$ and transition states $\left(\mathbf{T S}_{\mathbf{B} 1}\right.$ and $\left.\mathbf{T} \mathbf{S}_{\mathbf{B} 2}\right)$ with structural parameters.

\section{Calculations with the $(6,6)$ active space}

Energies at the optimized ground state, excited state and vertically excited states with $(6,6)$ active space calculations are shown in Table S1

Table S1: Absolute and relative energy values at CASSCF $(6,6)$ level and with dynamic correlation corrections on optimized structures of ground and excited states.

\begin{tabular}{|c|c|c|c|c|c|}
\hline \multirow[t]{2}{*}{ System } & \multirow[t]{2}{*}{$\begin{array}{l}\text { Molecular } \\
\text { States }\end{array}$} & \multicolumn{2}{|c|}{ CASSCF } & \multicolumn{2}{|c|}{$\begin{array}{c}\text { with dynamic correlation correction } \\
\text { (CASMP2) }\end{array}$} \\
\hline & & E(Hartree) & $\Delta \mathbf{E}(\mathrm{Kcal} / \mathrm{mol})$ & E(Hartree) & $\Delta E($ Kcal $/ \mathrm{mol})$ \\
\hline \multirow{3}{*}{ nitrone $A$} & $\mathbf{G S}_{\mathrm{A}}$ & -514.3449 & 0 & -515.9297 & 0 \\
\hline & $\mathbf{F C}_{\mathrm{A}}$ & -514.1875 & 98.77 & -515.7412 & 118.28 \\
\hline & $\mathbf{E S}_{\mathbf{A}}$ & -514.2283 & 73.17 & -515.7731 & 98.27 \\
\hline \multirow{3}{*}{ nitrone $B$} & $\mathbf{G S}_{\mathbf{B}}$ & -743.8904 & 0 & -746.2588 & 0 \\
\hline & $\mathbf{F C}_{\mathbf{B}}$ & -743.7296 & 100.90 & -746.0755 & 115.02 \\
\hline & $\mathbf{E S}_{\mathbf{B}}$ & -743.7909 & 62.43 & -746.1724 & 54.21 \\
\hline
\end{tabular}




\section{Conical intersection geometries at CASSCF $(6,6)$ level of calculations}

Our attempt to track oxaziridine structure from terminally-twisted conical intersection geometries was not successful. The vectors did not indicate any possibility of oxaziridine formation.

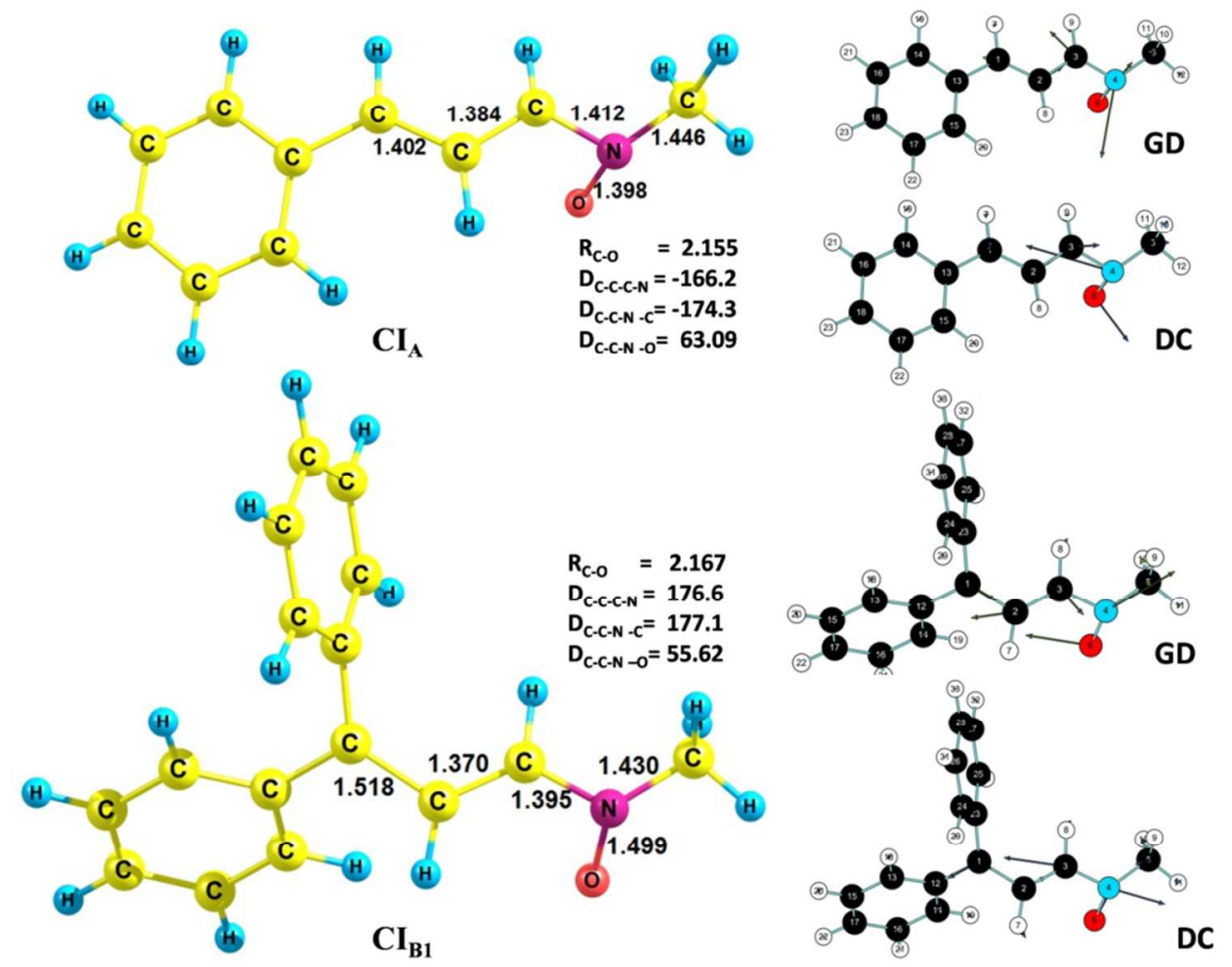

Figure S2: Conical intersection geometries of nitrone A and nitrone B at CASSCF $(6,6)$ level of calculations with their gradient difference (GD) and derivative coupling (DC) vectors. 


\section{Sloped Conical intersection of nitrone B}

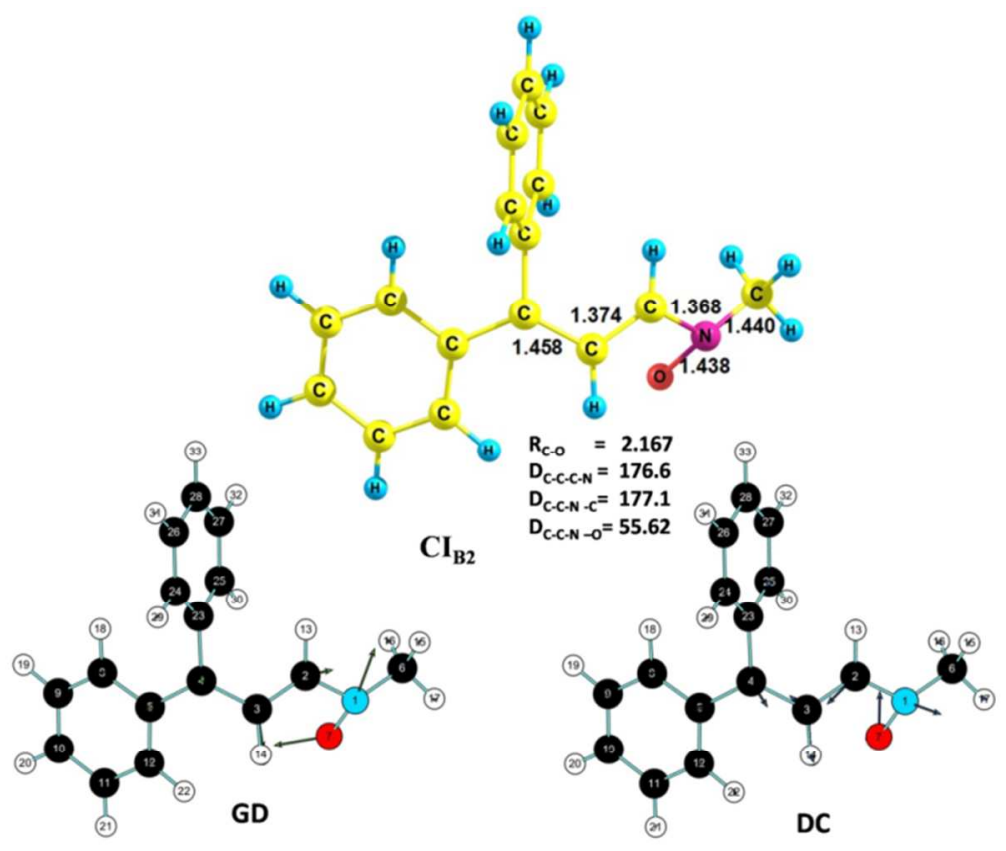

Figure S3: Conical intersection geometry $\left(\mathrm{CI}_{\mathrm{B} 2}\right)$ with its gradient difference $(\mathrm{GD})$ and derivative coupling (DC) vectors.

\section{Cartesian coordinates of important geometries.}

\section{a) Nitrone $\mathbf{A}$}

GS geometry of nitrone A at RHF level

$\begin{array}{rrrr}7 & 3.218687000 & -1.867129000 & 0.276218000 \\ 6 & 2.439221000 & -0.897537000 & -0.010881000 \\ 6 & 0.995793000 & -1.039968000 & 0.020239000 \\ 6 & 0.187705000 & -0.028117000 & -0.279333000 \\ 6 & -1.286120000 & -0.036700000 & -0.287297000 \\ 6 & 4.667938000 & -1.705826000 & 0.239658000 \\ 8 & 2.837517000 & -3.026588000 & 0.610220000 \\ 6 & -1.958931000 & 1.131263000 & -0.631320000 \\ 6 & -3.343084000 & 1.180206000 & -0.655360000 \\ 6 & -4.079760000 & 0.055856000 & -0.334007000 \\ 6 & -3.422121000 & -1.116873000 & 0.011295000 \\ 6 & -2.043250000 & -1.164205000 & 0.034830000 \\ 1 & 2.890423000 & 0.037347000 & -0.279169000 \\ 1 & 0.636993000 & -2.010186000 & 0.299441000 \\ 1 & 4.945782000 & -0.703231000 & -0.049524000 \\ 1 & 5.053230000 & -2.429050000 & -0.462976000 \\ 1 & 5.041252000 & -1.938764000 & 1.225343000\end{array}$




$\begin{array}{rrrr}1 & -1.395428000 & 2.012759000 & -0.883362000 \\ 1 & -3.840612000 & 2.094701000 & -0.924517000 \\ 1 & -5.154336000 & 0.088084000 & -0.350994000 \\ 1 & -3.987688000 & -1.996168000 & 0.262686000 \\ 1 & -1.557352000 & -2.082849000 & 0.305115000 \\ 1 & 0.631781000 & 0.917014000 & -0.550650000\end{array}$

GS geometry of nitrone A at DFT level

$\begin{array}{rrrr}7 & 3.225601000 & -1.902000000 & 0.286388000 \\ 6 & 2.434984000 & -0.884890000 & -0.014579000 \\ 6 & 1.013797000 & -1.007628000 & 0.010977000 \\ 6 & 0.176823000 & 0.011313000 & -0.290853000 \\ 6 & -1.281866000 & -0.014947000 & -0.293579000 \\ 6 & 4.692874000 & -1.737075000 & 0.248907000 \\ 8 & 2.828732000 & -3.060477000 & 0.619982000 \\ 6 & -1.983155000 & 1.154285000 & -0.638180000 \\ 6 & -3.373361000 & 1.184887000 & -0.656943000 \\ 6 & -4.100865000 & 0.043125000 & -0.330466000 \\ 6 & -3.422325000 & -1.127854000 & 0.014486000 \\ 6 & -2.035237000 & -1.159046000 & 0.033398000 \\ 1 & 2.920858000 & 0.044278000 & -0.280952000 \\ 1 & 0.642625000 & -1.986849000 & 0.292697000 \\ 1 & 4.972706000 & -0.725902000 & -0.042742000 \\ 1 & 5.084909000 & -2.465579000 & -0.460017000 \\ 1 & 5.072827000 & -1.971094000 & 1.242815000 \\ 1 & -1.423941000 & 2.049765000 & -0.894316000 \\ 1 & -3.888679000 & 2.101267000 & -0.926780000 \\ 1 & -5.185739000 & 0.061832000 & -0.343604000 \\ 1 & -3.981579000 & -2.022272000 & 0.270318000 \\ 1 & -1.530912000 & -2.079979000 & 0.304490000 \\ 1 & 0.618563000 & 0.968880000 & -0.565794000\end{array}$

GS geometry of nitrone A at CASSCF $(12,12)$ level

$\begin{array}{rrrr}7 & 3.237097000 & -1.879228000 & 0.279809000 \\ 6 & 2.451286000 & -0.894242000 & -0.011726000 \\ 6 & 1.010525000 & -1.030300000 & 0.017524000 \\ 6 & 0.182395000 & -0.007683000 & -0.285237000 \\ 6 & -1.290941000 & -0.024736000 & -0.290767000 \\ 6 & 4.688693000 & -1.725561000 & 0.245521000 \\ 8 & 2.858651000 & -3.055615000 & 0.618628000 \\ 6 & -1.979124000 & 1.142880000 & -0.634879000 \\ 6 & -3.370316000 & 1.179722000 & -0.655491000 \\ 6 & -4.099312000 & 0.056612000 & -0.334397000 \\ 6 & -3.435186000 & -1.120752000 & 0.012384000 \\ 6 & -2.048111000 & -1.163009000 & 0.034540000 \\ 1 & 2.916731000 & 0.032676000 & -0.277504000 \\ 1 & 0.639956000 & -1.996887000 & 0.295485000\end{array}$




$\begin{array}{rrrr}1 & 4.961172000 & -0.722351000 & -0.043779000 \\ 1 & 5.077388000 & -2.444022000 & -0.459999000 \\ 1 & 5.065361000 & -1.953083000 & 1.231109000 \\ 1 & -1.423536000 & 2.028916000 & -0.888334000 \\ 1 & -3.872835000 & 2.092053000 & -0.924120000 \\ 1 & -5.174280000 & 0.083769000 & -0.349941000 \\ 1 & -3.998373000 & -2.001463000 & 0.264246000 \\ 1 & -1.558022000 & -2.079846000 & 0.304408000 \\ 1 & 0.638419000 & 0.956191000 & -0.561826000\end{array}$

ES geometry of nitrone A at $\operatorname{CASSCF}(12,12)$ level

$\begin{array}{rrrr}7 & 3.501938000 & 0.120004000 & -0.158034000 \\ 6 & 2.320784000 & -0.604435000 & -0.082866000 \\ 6 & 1.049686000 & -0.013046000 & -0.051791000 \\ 6 & -0.137526000 & -0.810063000 & -0.044910000 \\ 6 & -1.453253000 & -0.356997000 & -0.016352000 \\ 6 & 4.777377000 & -0.479542000 & 0.168612000 \\ 8 & 3.429639000 & 1.419271000 & -0.020244000 \\ 6 & -2.554316000 & -1.311332000 & -0.010149000 \\ 6 & -3.859358000 & -0.902579000 & 0.019743000 \\ 6 & -4.186862000 & 0.491495000 & 0.045793000 \\ 6 & -3.135938000 & 1.448998000 & 0.039742000 \\ 6 & -1.820104000 & 1.058648000 & 0.009958000 \\ 1 & 2.448243000 & -1.668450000 & -0.121538000 \\ 1 & 0.996116000 & 1.055257000 & -0.030734000 \\ 1 & 4.839689000 & -1.460820000 & -0.283595000 \\ 1 & 4.914718000 & -0.573388000 & 1.241840000 \\ 1 & 5.556431000 & 0.147582000 & -0.237531000 \\ 1 & -2.318521000 & -2.360815000 & -0.029721000 \\ 1 & -4.650918000 & -1.630325000 & 0.023640000 \\ 1 & -5.212244000 & 0.808100000 & 0.069154000 \\ 1 & -3.378925000 & 2.496520000 & 0.058499000 \\ 1 & -1.048240000 & 1.803664000 & 0.005188000 \\ 1 & 0.002534000 & -1.878897000 & -0.063132000\end{array}$

GS geometry of nitrone A at $\operatorname{CASSCF}(6,6)$ level

$\begin{array}{lrrc}7 & 3.417090000 & 0.133065000 & 0.000036000 \\ 6 & 2.350833000 & -0.614033000 & -0.000076000 \\ 6 & 1.017339000 & -0.033514000 & -0.000003000 \\ 6 & -0.075485000 & -0.789455000 & -0.000122000 \\ 6 & -1.477326000 & -0.331923000 & -0.000066000 \\ 6 & 4.752677000 & -0.455025000 & -0.000034000 \\ 8 & 3.405451000 & 1.399241000 & 0.000211000 \\ 6 & -2.485094000 & -1.272024000 & 0.000076000 \\ 6 & -3.833836000 & -0.889161000 & 0.000149000 \\ 6 & -4.168315000 & 0.447546000 & 0.000069000 \\ 6 & -3.148014000 & 1.413754000 & -0.000091000\end{array}$




$\begin{array}{rrrc}6 & -1.834643000 & 1.034047000 & -0.000159000 \\ 1 & 2.493547000 & -1.676140000 & -0.000221000 \\ 1 & 0.985352000 & 1.037508000 & 0.000159000 \\ 1 & 4.707646000 & -1.534374000 & -0.000183000 \\ 1 & 5.263630000 & -0.094668000 & 0.879954000 \\ 1 & 5.263644000 & -0.094426000 & -0.879914000 \\ 1 & -2.236853000 & -2.319186000 & 0.000141000 \\ 1 & -4.600927000 & -1.642768000 & 0.000266000 \\ 1 & -5.199338000 & 0.752586000 & 0.000120000 \\ 1 & -3.402635000 & 2.458513000 & -0.000166000 \\ 1 & -1.069982000 & 1.787996000 & -0.000297000 \\ 1 & 0.043860000 & -1.861684000 & -0.000260000\end{array}$

ES geometry of nitrone A at $\operatorname{CASSCF}(6,6)$ level

$\begin{array}{rrrr}7 & 3.296651000 & -1.858680000 & 0.493811000 \\ 6 & 2.406775000 & -0.860753000 & 0.076874000 \\ 6 & 1.011932000 & -1.026003000 & 0.099669000 \\ 6 & 0.131139000 & 0.035373000 & -0.274722000 \\ 6 & -1.261353000 & 0.003179000 & -0.295939000 \\ 6 & 4.707539000 & -1.777466000 & 0.179362000 \\ 8 & 2.834320000 & -2.996328000 & 0.802433000 \\ 6 & -2.013290000 & 1.182619000 & -0.710690000 \\ 6 & -3.355212000 & 1.189714000 & -0.746561000 \\ 6 & -4.103413000 & 0.027136000 & -0.374430000 \\ 6 & -3.395309000 & -1.142763000 & 0.037105000 \\ 6 & -2.051779000 & -1.166396000 & 0.078476000 \\ 1 & 2.868650000 & 0.066149000 & -0.199653000 \\ 1 & 0.630619000 & -1.977910000 & 0.405270000 \\ 1 & 5.061379000 & -0.767661000 & 0.343093000 \\ 1 & 4.901767000 & -2.059169000 & -0.851417000 \\ 1 & 5.232581000 & -2.451263000 & 0.839015000 \\ 1 & -1.459617000 & 2.061413000 & -0.992170000 \\ 1 & -3.882827000 & 2.074186000 & -1.056884000 \\ 1 & -5.175828000 & 0.034718000 & -0.403750000 \\ 1 & -3.954876000 & -2.017550000 & 0.318061000 \\ 1 & -1.546958000 & -2.059929000 & 0.392665000 \\ 1 & 0.594751000 & 0.961425000 & -0.573959000\end{array}$

$\mathrm{GS}_{\mathrm{A}}$ geometry of nitrone A at $\operatorname{CASSCF}(4,4)$ level

$\begin{array}{lrrc}7 & 3.419939000 & 0.131814000 & 0.000621000 \\ 6 & 2.351666000 & -0.612798000 & -0.001290000 \\ 6 & 1.019827000 & -0.028485000 & 0.000005000 \\ 6 & -0.075100000 & -0.781437000 & -0.002036000 \\ 6 & -1.476262000 & -0.319864000 & -0.001104000 \\ 6 & 4.754003000 & -0.459120000 & -0.000702000 \\ 8 & 3.411060000 & 1.398316000 & 0.003694000 \\ 6 & -2.493124000 & -1.270633000 & 0.001377000 \\ 6 & -3.826566000 & -0.894184000 & 0.002539000\end{array}$




$\begin{array}{rrrr}6 & -4.166585000 & 0.446404000 & 0.001093000 \\ 6 & -3.163374000 & 1.406231000 & -0.001578000 \\ 6 & -1.834724000 & 1.029686000 & -0.002721000 \\ 1 & 2.491908000 & -1.675202000 & -0.003971000 \\ 1 & 0.990704000 & 1.042606000 & 0.002833000 \\ 1 & 4.705802000 & -1.538305000 & -0.003975000 \\ 1 & 5.265687000 & -0.102864000 & 0.880519000 \\ 1 & 5.266271000 & -0.097540000 & -0.879406000 \\ 1 & -2.239286000 & -2.316502000 & 0.002523000 \\ 1 & -4.594219000 & -1.647266000 & 0.004541000 \\ 1 & -5.199743000 & 0.744233000 & 0.001925000 \\ 1 & -3.419107000 & 2.450734000 & -0.002879000 \\ 1 & -1.076239000 & 1.790024000 & -0.005084000 \\ 1 & 0.041603000 & -1.853939000 & -0.004421000\end{array}$

$\mathrm{ES}_{\mathrm{A}}$ geometry of nitrone A at $\operatorname{CASSCF}(4,4)$ level

$\begin{array}{rrrr}7 & -3.441685000 & 0.194776000 & 0.174806000 \\ 6 & -2.304510000 & -0.610816000 & 0.112228000 \\ 6 & -1.048225000 & -0.108715000 & -0.383822000 \\ 6 & 0.156633000 & -0.925602000 & -0.241105000 \\ 6 & 1.497198000 & -0.394188000 & -0.093293000 \\ 6 & -4.752419000 & -0.391789000 & 0.366525000 \\ 8 & -3.399981000 & 1.321660000 & -0.413988000 \\ 6 & 2.595397000 & -1.263265000 & -0.108310000 \\ 6 & 3.883520000 & -0.786392000 & 0.030270000 \\ 6 & 4.111568000 & 0.573277000 & 0.188946000 \\ 6 & 3.036245000 & 1.446742000 & 0.209262000 \\ 6 & 1.743875000 & 0.973015000 & 0.069956000 \\ 1 & -2.366053000 & -1.530862000 & 0.658520000 \\ 1 & -1.043871000 & 0.795267000 & -0.957391000 \\ 1 & -4.699811000 & -1.154410000 & 1.132717000 \\ 1 & -5.128955000 & -0.833655000 & -0.551059000 \\ 1 & -5.422749000 & 0.391078000 & 0.687605000 \\ 1 & 2.429063000 & -2.319229000 & -0.233006000 \\ 1 & 4.711335000 & -1.472976000 & 0.014272000 \\ 1 & 5.114597000 & 0.945528000 & 0.296123000 \\ 1 & 3.203396000 & 2.501710000 & 0.335831000 \\ 1 & 0.921622000 & 1.663443000 & 0.098453000 \\ 1 & 0.057371000 & -1.996207000 & -0.297740000\end{array}$

$\mathrm{TS}_{\mathrm{A}}$ geometry of nitrone A at $\operatorname{CASSCF}(4,4)$ level

$\begin{array}{rrrr}7 & -3.482972000 & 0.139778000 & 0.010169000 \\ 6 & -2.304567000 & -0.592368000 & 0.141468000 \\ 6 & -1.041134000 & -0.095364000 & -0.341638000 \\ 6 & 0.165523000 & -0.919268000 & -0.234965000 \\ 6 & 1.507160000 & -0.390011000 & -0.088122000 \\ 6 & -4.748044000 & -0.359679000 & 0.501744000\end{array}$




$\begin{array}{rrrr}8 & -3.442482000 & 1.280915000 & -0.540398000 \\ 6 & 2.606765000 & -1.255200000 & -0.150282000 \\ 6 & 3.895913000 & -0.781066000 & -0.010977000 \\ 6 & 4.124440000 & 0.572108000 & 0.194826000 \\ 6 & 3.047911000 & 1.441665000 & 0.261618000 \\ 6 & 1.754486000 & 0.970566000 & 0.122654000 \\ 1 & -2.380202000 & -1.486747000 & 0.726131000 \\ 1 & -1.036285000 & 0.811482000 & -0.911604000 \\ 1 & -4.750662000 & -0.421447000 & 1.585735000 \\ 1 & -4.956937000 & -1.342933000 & 0.094242000 \\ 1 & -5.511841000 & 0.330627000 & 0.181010000 \\ 1 & 2.440652000 & -2.306129000 & -0.312212000 \\ 1 & 4.724362000 & -1.465100000 & -0.063696000 \\ 1 & 5.128249000 & 0.942199000 & 0.302194000 \\ 1 & 3.214836000 & 2.491726000 & 0.424465000 \\ 1 & 0.931918000 & 1.658322000 & 0.187316000 \\ 1 & 0.065844000 & -1.986062000 & -0.339548000\end{array}$

$\mathrm{OX}_{\mathrm{A}}$ geometry of nitrone A at $\mathrm{CASSCF}(4,4)$ level

$\begin{array}{rrrr}6 & -0.001611000 & 0.549940000 & -0.324207000 \\ 6 & 1.029451000 & -0.245836000 & -0.090011000 \\ 6 & 2.427213000 & 0.184108000 & -0.317103000 \\ 7 & 3.429423000 & -0.334125000 & 0.519535000 \\ 6 & 4.614777000 & 0.483839000 & 0.679799000 \\ 8 & 3.302608000 & -0.740494000 & -0.857953000 \\ 1 & 0.196394000 & 1.548371000 & -0.681900000 \\ 1 & 0.918416000 & -1.246043000 & 0.286904000 \\ 1 & 2.551537000 & 1.190007000 & -0.689415000 \\ 1 & 4.777629000 & 1.159075000 & -0.154567000 \\ 1 & 5.488829000 & -0.181569000 & 0.777436000 \\ 1 & 4.506040000 & 1.048377000 & 1.598740000 \\ 6 & -1.433179000 & 0.235403000 & -0.132494000 \\ 6 & -1.906090000 & -1.068492000 & 0.014917000 \\ 6 & -2.353411000 & 1.279013000 & -0.103703000 \\ 6 & -3.253226000 & -1.313018000 & 0.202423000 \\ 6 & -3.703988000 & 1.035736000 & 0.085591000 \\ 6 & -4.158292000 & -0.261883000 & 0.241326000 \\ 1 & -1.223401000 & -1.896718000 & -0.030735000 \\ 1 & -2.009569000 & 2.291610000 & -0.224938000 \\ 1 & -3.599685000 & -2.325149000 & 0.311826000 \\ 1 & -4.396806000 & 1.857810000 & 0.109160000 \\ 1 & -5.206063000 & -0.455807000 & 0.385138000\end{array}$




\section{b) Nitrone B}

GS geometry of nitrone B at RHF level

$\begin{array}{rrrr}7 & 3.106211000 & -1.898824000 & 0.504500000 \\ 6 & 2.459751000 & -0.899613000 & 0.038807000 \\ 6 & 1.009398000 & -0.846417000 & 0.108618000 \\ 6 & 0.260219000 & 0.181601000 & -0.295698000 \\ 6 & -1.228780000 & 0.080303000 & -0.285321000 \\ 6 & 4.559399000 & -1.967887000 & 0.396215000 \\ 8 & 2.586604000 & -2.895975000 & 1.083228000 \\ 6 & -2.011658000 & 1.180714000 & 0.056857000 \\ 6 & -3.390836000 & 1.083758000 & 0.102016000 \\ 6 & -4.016882000 & -0.111322000 & -0.209954000 \\ 6 & -3.251840000 & -1.208016000 & -0.568704000 \\ 6 & -1.872035000 & -1.112692000 & -0.607834000 \\ 1 & 3.027187000 & -0.123067000 & -0.428579000 \\ 1 & 0.550788000 & -1.731227000 & 0.502132000 \\ 1 & 4.959274000 & -1.096432000 & -0.100005000 \\ 1 & 4.797566000 & -2.865998000 & -0.153274000 \\ 1 & 4.951779000 & -2.054338000 & 1.398087000 \\ 1 & -1.540819000 & 2.115181000 & 0.299465000 \\ 1 & -3.975355000 & 1.942378000 & 0.380485000 \\ 1 & -5.089229000 & -0.185077000 & -0.180489000 \\ 1 & -3.727555000 & -2.137467000 & -0.825832000 \\ 1 & -1.292861000 & -1.966045000 & -0.907848000 \\ 6 & 0.854541000 & 1.473513000 & -0.765326000 \\ 6 & 1.635186000 & 2.252823000 & 0.079798000 \\ 6 & 0.618421000 & 1.925259000 & -2.061174000 \\ 6 & 2.180120000 & 3.449303000 & -0.361885000 \\ 6 & 1.169472000 & 3.112707000 & -2.505800000 \\ 6 & 1.952509000 & 3.879731000 & -1.656267000 \\ 1 & 1.806519000 & 1.928107000 & 1.090367000 \\ 1 & 0.003850000 & 1.340793000 & -2.721883000 \\ 1 & 2.776361000 & 4.042847000 & 0.307972000 \\ 1 & 0.985591000 & 3.441913000 & -3.512825000 \\ 1 & 2.374964000 & 4.806642000 & -2.000573000\end{array}$

GS geometry of nitrone B at DFT level

$\begin{array}{rrrr}7 & 3.105236000 & -1.939861000 & 0.538410000 \\ 6 & 2.452990000 & -0.893066000 & 0.055823000 \\ 6 & 1.028615000 & -0.824335000 & 0.120487000 \\ 6 & 0.251948000 & 0.212031000 & -0.300456000 \\ 6 & -1.224304000 & 0.085712000 & -0.306138000 \\ 6 & 4.574011000 & -2.020602000 & 0.404802000 \\ 8 & 2.570501000 & -2.928943000 & 1.126443000 \\ 6 & -2.042578000 & 1.204857000 & -0.072768000 \\ 6 & -3.428055000 & 1.086036000 & -0.037122000 \\ 6 & -4.033785000 & -0.151380000 & -0.245763000\end{array}$




$\begin{array}{rrrr}6 & -3.238546000 & -1.269235000 & -0.494186000 \\ 6 & -1.853691000 & -1.152456000 & -0.527333000 \\ 1 & 3.051194000 & -0.127896000 & -0.415295000 \\ 1 & 0.559584000 & -1.712136000 & 0.529300000 \\ 1 & 4.977213000 & -1.139153000 & -0.091452000 \\ 1 & 4.801702000 & -2.921393000 & -0.164559000 \\ 1 & 4.987324000 & -2.122821000 & 1.407672000 \\ 1 & -1.585070000 & 2.172583000 & 0.099292000 \\ 1 & -4.036466000 & 1.963723000 & 0.157548000 \\ 1 & -5.114937000 & -0.242871000 & -0.223213000 \\ 1 & -3.699295000 & -2.235448000 & -0.674198000 \\ 1 & -1.251288000 & -2.025578000 & -0.752501000 \\ 6 & 0.842806000 & 1.501363000 & -0.757032000 \\ 6 & 1.730617000 & 2.221659000 & 0.055744000 \\ 6 & 0.515324000 & 2.031612000 & -2.014663000 \\ 6 & 2.284067000 & 3.424142000 & -0.379129000 \\ 6 & 1.075884000 & 3.226941000 & -2.453324000 \\ 6 & 1.962926000 & 3.928337000 & -1.637108000 \\ 1 & 1.968801000 & 1.842702000 & 1.044069000 \\ 1 & -0.178210000 & 1.493931000 & -2.652717000 \\ 1 & 2.960851000 & 3.970780000 & 0.270189000 \\ 1 & 0.818483000 & 3.613419000 & -3.434466000 \\ 1 & 2.394009000 & 4.864520000 & -1.977081000\end{array}$

GS geometry of nitrone B at $\operatorname{CASSCF}(6,6)$ level

$\begin{array}{rrrr}7 & 3.115142000 & -1.914362000 & 0.550901000 \\ 6 & 2.471545000 & -0.891510000 & 0.062336000 \\ 6 & 1.017123000 & -0.832240000 & 0.121895000 \\ 6 & 0.270219000 & 0.192083000 & -0.293785000 \\ 6 & -1.220927000 & 0.081249000 & -0.288992000 \\ 6 & 4.569252000 & -2.002029000 & 0.456505000 \\ 8 & 2.573191000 & -2.896947000 & 1.134424000 \\ 6 & -2.016124000 & 1.166757000 & 0.132766000 \\ 6 & -3.381656000 & 1.062224000 & 0.168341000 \\ 6 & -4.012197000 & -0.124663000 & -0.228383000 \\ 6 & -3.255253000 & -1.185019000 & -0.653663000 \\ 6 & -1.857488000 & -1.085083000 & -0.687238000 \\ 1 & 3.052536000 & -0.129878000 & -0.412225000 \\ 1 & 0.552238000 & -1.713786000 & 0.517166000 \\ 1 & 4.983515000 & -1.139407000 & -0.044435000 \\ 1 & 4.803422000 & -2.906361000 & -0.084620000 \\ 1 & 4.953624000 & -2.081018000 & 1.462179000 \\ 1 & -1.542533000 & 2.081850000 & 0.437699000 \\ 1 & -3.973321000 & 1.895913000 & 0.502521000 \\ 1 & -5.084846000 & -0.197295000 & -0.202776000 \\ 1 & -3.727220000 & -2.098060000 & -0.970057000 \\ 1 & -1.276253000 & -1.916604000 & -1.041294000 \\ 6 & 0.854674000 & 1.482670000 & -0.778102000\end{array}$




$\begin{array}{rrrr}6 & 1.678918000 & 2.251528000 & 0.037354000 \\ 6 & 0.563659000 & 1.948160000 & -2.059330000 \\ 6 & 2.213599000 & 3.447105000 & -0.419919000 \\ 6 & 1.102706000 & 3.136055000 & -2.518949000 \\ 6 & 1.930696000 & 3.890001000 & -1.699946000 \\ 1 & 1.890906000 & 1.919063000 & 1.037774000 \\ 1 & -0.085019000 & 1.373902000 & -2.696167000 \\ 1 & 2.844137000 & 4.030885000 & 0.226830000 \\ 1 & 0.875176000 & 3.475483000 & -3.513653000 \\ 1 & 2.344422000 & 4.816509000 & -2.055884000\end{array}$

ES geometry of nitrone B at $\operatorname{CASSCF}(6,6)$ level

$\begin{array}{rrrr}7 & 3.435647000 & -0.992868000 & 1.443108000 \\ 6 & 2.564354000 & -0.336992000 & 0.555408000 \\ 6 & 1.236782000 & -0.277970000 & 0.650973000 \\ 6 & 0.371353000 & 0.484513000 & -0.232604000 \\ 6 & -1.060674000 & 0.135686000 & -0.305207000 \\ 6 & 4.379419000 & -1.892086000 & 0.789634000 \\ 8 & 2.710767000 & -1.782896000 & 2.332270000 \\ 6 & -2.028007000 & 1.108151000 & -0.578425000 \\ 6 & -3.371825000 & 0.785618000 & -0.621889000 \\ 6 & -3.788814000 & -0.517408000 & -0.398444000 \\ 6 & -2.844566000 & -1.494505000 & -0.131966000 \\ 6 & -1.498946000 & -1.173308000 & -0.086020000 \\ 1 & 3.104170000 & 0.191241000 & -0.206856000 \\ 1 & 0.750346000 & -0.834995000 & 1.430261000 \\ 1 & 4.976789000 & -1.306260000 & 0.104423000 \\ 1 & 3.875093000 & -2.689722000 & 0.251513000 \\ 1 & 5.033693000 & -2.314527000 & 1.540106000 \\ 1 & -1.724117000 & 2.124591000 & -0.745606000 \\ 1 & -4.095335000 & 1.555102000 & -0.825529000 \\ 1 & -4.834187000 & -0.766972000 & -0.433679000 \\ 1 & -3.152982000 & -2.511491000 & 0.034337000 \\ 1 & -0.781589000 & -1.950846000 & 0.101962000 \\ 6 & 0.911080000 & 1.581475000 & -1.075652000 \\ 6 & 1.694971000 & 2.596705000 & -0.525909000 \\ 6 & 0.641586000 & 1.628070000 & -2.444038000 \\ 6 & 2.192270000 & 3.619637000 & -1.315762000 \\ 6 & 1.143166000 & 2.647478000 & -3.234872000 \\ 6 & 1.920643000 & 3.648075000 & -2.673999000 \\ 1 & 1.906791000 & 2.587145000 & 0.527649000 \\ 1 & 0.037591000 & 0.858098000 & -2.888718000 \\ 1 & 2.787331000 & 4.395908000 & -0.868287000 \\ 1 & 0.927629000 & 2.658846000 & -4.288584000 \\ 1 & 2.308066000 & 4.441652000 & -3.287701000\end{array}$

$\mathrm{GS}_{\mathrm{B}}$ geometry of nitrone B at CASSCF $(4,4)$ level $\begin{array}{llll}7 & 3.113496000 & -1.914788000 & 0.526946000\end{array}$ 


$\begin{array}{rrrr}6 & 2.465808000 & -0.890210000 & 0.047810000 \\ 6 & 1.011958000 & -0.834464000 & 0.112591000 \\ 6 & 0.262713000 & 0.192257000 & -0.294972000 \\ 6 & -1.227086000 & 0.085608000 & -0.285629000 \\ 6 & 4.567459000 & -1.998375000 & 0.427962000 \\ 8 & 2.575150000 & -2.903513000 & 1.104501000 \\ 6 & -2.014894000 & 1.174924000 & 0.084155000 \\ 6 & -3.394036000 & 1.071483000 & 0.124720000 \\ 6 & -4.015359000 & -0.119012000 & -0.217884000 \\ 6 & -3.245635000 & -1.204124000 & -0.60238900 \\ 6 & -1.865185000 & -1.101947000 & -0.638334000 \\ 1 & 3.043621000 & -0.123211000 & -0.421945000 \\ 1 & 0.550319000 & -1.718279000 & 0.505933000 \\ 1 & 4.978010000 & -1.133811000 & -0.072449000 \\ 1 & 4.802044000 & -2.900991000 & -0.115623000 \\ 1 & 4.955103000 & -2.078597000 & 1.432212000 \\ 1 & -1.546210000 & 2.104160000 & 0.351046000 \\ 1 & -3.982782000 & 1.920324000 & 0.423697000 \\ 1 & -5.087500000 & -0.197416000 & -0.191477000 \\ 1 & -3.717631000 & -2.128981000 & -0.882117000 \\ 1 & -1.280282000 & -1.944714000 & -0.957603000 \\ 6 & 0.852046000 & 1.484256000 & -0.770926000 \\ 6 & 1.658123000 & 2.256267000 & 0.059185000 \\ 6 & 0.586902000 & 1.945416000 & -2.059444000 \\ 6 & 2.199332000 & 3.452167000 & -0.389878000 \\ 6 & 1.133049000 & 3.133127000 & -2.510994000 \\ 6 & 1.941962000 & 3.891200000 & -1.676587000 \\ 1 & 1.851594000 & 1.925303000 & 1.063839000 \\ 1 & -0.047148000 & 1.367549000 & -2.707526000 \\ 1 & 2.815657000 & 4.038597000 & 0.268006000 \\ 1 & 0.926163000 & 3.469334000 & -3.511261000 \\ 1 & 2.361101000 & 4.817635000 & -2.026291000\end{array}$

$\mathrm{ES}_{\mathrm{B}}$ geometry of nitrone B at CASSCF $(4,4)$ level

$\begin{array}{rrrr}7 & 3.435647000 & -0.992868000 & 1.443108000 \\ 6 & 2.564354000 & -0.336992000 & 0.555408000 \\ 6 & 1.236782000 & -0.277970000 & 0.650973000 \\ 6 & 0.371353000 & 0.484513000 & -0.232604000 \\ 6 & -1.060674000 & 0.135686000 & -0.305207000 \\ 6 & 4.379419000 & -1.892086000 & 0.789634000 \\ 8 & 2.710767000 & -1.782896000 & 2.332270000 \\ 6 & -2.028007000 & 1.108151000 & -0.578425000 \\ 6 & -3.371825000 & 0.785618000 & -0.621889000 \\ 6 & -3.788814000 & -0.517408000 & -0.398444000 \\ 6 & -2.844566000 & -1.494505000 & -0.131966000 \\ 6 & -1.498946000 & -1.173308000 & -0.086020000 \\ 1 & 3.104170000 & 0.191241000 & -0.206856000 \\ 1 & 0.750346000 & -0.834995000 & 1.430261000\end{array}$




$\begin{array}{rrrr}1 & 4.976789000 & -1.306260000 & 0.104423000 \\ 1 & 3.875093000 & -2.689722000 & 0.251513000 \\ 1 & 5.033693000 & -2.314527000 & 1.540106000 \\ 1 & -1.724117000 & 2.124591000 & -0.745606000 \\ 1 & -4.095335000 & 1.555102000 & -0.825529000 \\ 1 & -4.834187000 & -0.766972000 & -0.433679000 \\ 1 & -3.152982000 & -2.511491000 & 0.034337000 \\ 1 & -0.781589000 & -1.950846000 & 0.101962000 \\ 6 & 0.911080000 & 1.581475000 & -1.075652000 \\ 6 & 1.694971000 & 2.596705000 & -0.525909000 \\ 6 & 0.641586000 & 1.628070000 & -2.444038000 \\ 6 & 2.192270000 & 3.619637000 & -1.315762000 \\ 6 & 1.143166000 & 2.647478000 & -3.234872000 \\ 6 & 1.920643000 & 3.648075000 & -2.673999000 \\ 1 & 1.906791000 & 2.587145000 & 0.527649000 \\ 1 & 0.037591000 & 0.858098000 & -2.888718000 \\ 1 & 2.787331000 & 4.395908000 & -0.868287000 \\ 1 & 0.927629000 & 2.658846000 & -4.288584000 \\ 1 & 2.308066000 & 4.441652000 & -3.287701000\end{array}$

SP geometry of nitrone B at CASSCF $(4,4)$ level

$\begin{array}{rrrr}7 & 3.157344000 & -1.683526000 & 1.041077000 \\ 6 & 2.374164000 & -0.805079000 & 0.279842000 \\ 6 & 1.054584000 & -0.669808000 & 0.424847000 \\ 6 & 0.225565000 & 0.251961000 & -0.321792000 \\ 6 & -1.211246000 & 0.097611000 & -0.332513000 \\ 6 & 4.534044000 & -1.958014000 & 0.682061000 \\ 8 & 2.614950000 & -2.436135000 & 1.893237000 \\ 6 & -2.107323000 & 1.217712000 & -0.660151000 \\ 6 & -3.434169000 & 1.092453000 & -0.615664000 \\ 6 & -4.059137000 & -0.170592000 & -0.249447000 \\ 6 & -3.190329000 & -1.297525000 & 0.054753000 \\ 6 & -1.862883000 & -1.174139000 & 0.020204000 \\ 1 & 2.940738000 & -0.235862000 & -0.429873000 \\ 1 & 0.576988000 & -1.298189000 & 1.151411000 \\ 1 & 5.025674000 & -1.043238000 & 0.377218000 \\ 1 & 4.593792000 & -2.679086000 & -0.127507000 \\ 1 & 5.027704000 & -2.358001000 & 1.554176000 \\ 1 & -1.667126000 & 2.161944000 & -0.917518000 \\ 1 & -4.063911000 & 1.934055000 & -0.843128000 \\ 1 & -5.125447000 & -0.267060000 & -0.203171000 \\ 1 & -3.637972000 & -2.244501000 & 0.298680000 \\ 1 & -1.246756000 & -2.029752000 & 0.221433000 \\ 6 & 0.857577000 & 1.350908000 & -1.105814000 \\ 6 & 1.678866000 & 2.294572000 & -0.489923000 \\ 6 & 0.639130000 & 1.462682000 & -2.477946000 \\ 6 & 2.261148000 & 3.316219000 & -1.221454000\end{array}$




$\begin{array}{llll}6 & 1.224744000 & 2.481146000 & -3.211505000 \\ 6 & 2.037767000 & 3.412165000 & -2.585711000 \\ 1 & 1.853215000 & 2.229657000 & 0.568886000 \\ 1 & 0.008825000 & 0.744546000 & -2.971224000 \\ 1 & 2.884447000 & 4.038744000 & -0.725025000 \\ 1 & 1.046622000 & 2.545702000 & -4.270237000 \\ 1 & 2.490654000 & 4.204762000 & -3.154191000\end{array}$

$\mathrm{TS}_{\mathrm{B} 1}$ geometry of nitrone $\mathrm{B}$ at $\mathrm{CASSCF}(4,4)$ level

$\begin{array}{rrrr}7 & 3.169941000 & -2.241752000 & -0.205426000 \\ 6 & 2.430978000 & -1.054451000 & -0.072087000 \\ 6 & 1.155957000 & -1.128765000 & -0.393891000 \\ 6 & 0.264962000 & 0.009261000 & -0.575334000 \\ 6 & -1.198846000 & -0.151328000 & -0.623757000 \\ 6 & 4.536280000 & -2.214824000 & 0.250840000 \\ 8 & 3.220800000 & -2.272673000 & -1.666516000 \\ 6 & -2.010450000 & 0.833358000 & -1.203210000 \\ 6 & -3.381005000 & 0.670995000 & -1.304536000 \\ 6 & -3.992744000 & -0.476492000 & -0.829456000 \\ 6 & -3.210404000 & -1.460087000 & -0.246456000 \\ 6 & -1.840139000 & -1.302130000 & -0.142985000 \\ 1 & 2.949181000 & -0.143663000 & 0.195490000 \\ 1 & 0.804375000 & -2.117370000 & -0.648274000 \\ 1 & 4.488656000 & -2.020860000 & 1.319103000 \\ 1 & 5.114644000 & -1.437627000 & -0.240817000 \\ 1 & 4.994337000 & -3.179304000 & 0.085843000 \\ 1 & -1.566120000 & 1.723651000 & -1.604806000 \\ 1 & -3.970690000 & 1.441988000 & -1.769045000 \\ 1 & -5.057583000 & -0.603664000 & -0.912468000 \\ 1 & -3.667035000 & -2.358220000 & 0.131796000 \\ 1 & -1.277358000 & -2.087894000 & 0.324057000 \\ 6 & 0.876416000 & 1.334396000 & -0.913051000 \\ 6 & 0.725666000 & 2.449478000 & -0.095707000 \\ 6 & 1.639994000 & 1.457881000 & -2.073858000 \\ 6 & 1.299264000 & 3.665711000 & -0.443998000 \\ 6 & 2.210880000 & 2.671704000 & -2.421269000 \\ 6 & 2.038134000 & 3.781322000 & -1.608035000 \\ 1 & 0.146502000 & 2.374867000 & 0.808027000 \\ 1 & 1.791640000 & 0.599269000 & -2.703840000 \\ 1 & 1.166321000 & 4.520238000 & 0.195661000 \\ 1 & 2.793420000 & 2.747642000 & -3.322543000 \\ 1 & 2.481373000 & 4.723447000 & -1.877891000\end{array}$

$\mathrm{TS}_{\mathrm{B} 2}$ geometry of nitrone B at CASSCF $(4,4)$ level

$\begin{array}{llll}7 & 2.952478000 & -1.846672000 & 1.169455000 \\ 6 & 1.982193000 & -1.238201000 & 0.357831000 \\ 6 & 1.497681000 & -0.023288000 & 0.551009000\end{array}$




$\begin{array}{rrrr}6 & 0.480463000 & 0.617423000 & -0.317125000 \\ 6 & -0.911477000 & 0.160830000 & -0.368518000 \\ 6 & 3.663704000 & -3.028586000 & 0.723752000 \\ 8 & 3.414605000 & -1.229765000 & 2.164150000 \\ 6 & -1.979168000 & 1.028137000 & -0.876529000 \\ 6 & -3.246713000 & 0.613079000 & -0.955763000 \\ 6 & -3.628142000 & -0.719907000 & -0.524411000 \\ 6 & -2.600056000 & -1.557499000 & 0.066413000 \\ 6 & -1.331578000 & -1.144289000 & 0.146021000 \\ 1 & 1.654600000 & -1.864521000 & -0.450788000 \\ 1 & 1.908272000 & 0.556417000 & 1.359510000 \\ 1 & 2.980017000 & -3.706572000 & 0.229333000 \\ 1 & 4.079480000 & -3.510249000 & 1.595185000 \\ 1 & 4.464953000 & -2.770683000 & 0.038234000 \\ 1 & -1.726368000 & 2.024923000 & -1.181156000 \\ 1 & -4.007240000 & 1.274712000 & -1.330628000 \\ 1 & -4.641549000 & -1.059572000 & -0.603610000 \\ 1 & -2.876114000 & -2.523336000 & 0.450890000 \\ 1 & -0.597674000 & -1.776167000 & 0.604704000 \\ 6 & 0.924669000 & 1.818575000 & -1.075734000 \\ 6 & 1.633711000 & 2.846234000 & -0.455438000 \\ 6 & 0.681852000 & 1.924752000 & -2.445658000 \\ 6 & 2.076418000 & 3.943117000 & -1.175722000 \\ 6 & 1.126989000 & 3.018965000 & -3.166803000 \\ 6 & 1.826046000 & 4.034710000 & -2.534579000 \\ 1 & 1.835468000 & 2.796752000 & 0.599197000 \\ 1 & 0.145587000 & 1.140388000 & -2.949230000 \\ 1 & 2.615702000 & 4.726133000 & -0.672824000 \\ 1 & 0.931473000 & 3.075121000 & -4.223056000 \\ 1 & 2.171962000 & 4.885370000 & -3.094084000\end{array}$

$\mathrm{OX}_{\mathrm{B}}$ geometry of nitrone $\mathrm{B}$ at $\mathrm{CASSCF}(4,4)$ level

$\begin{array}{rrrr}6 & -0.721844000 & 0.855568000 & 2.896097000 \\ 6 & -1.992864000 & 0.526518000 & 2.523445000 \\ 6 & -2.371105000 & -0.275155000 & 1.332935000 \\ 7 & -3.397419000 & -1.224964000 & 1.479294000 \\ 6 & -3.386104000 & -2.326216000 & 0.536541000 \\ 8 & -3.603462000 & 0.013198000 & 0.771838000 \\ 1 & -2.828174000 & 0.871235000 & 3.104711000 \\ 1 & -1.593926000 & -0.481771000 & 0.617435000 \\ 1 & -2.867751000 & -2.084430000 & -0.386174000 \\ 1 & -4.408496000 & -2.596694000 & 0.309419000 \\ 1 & -2.902543000 & -3.168056000 & 1.019167000 \\ 6 & -0.494762000 & 1.760047000 & 4.067318000 \\ 6 & -1.271849000 & 2.899941000 & 4.251756000 \\ 6 & 0.500401000 & 1.477044000 & 5.000022000 \\ 6 & -1.072647000 & 3.724857000 & 5.347031000 \\ 6 & 0.696510000 & 2.297414000 & 6.097028000 \\ 6 & -0.090075000 & 3.424736000 & 6.274978000\end{array}$




$\begin{array}{rrrr}1 & -2.026977000 & 3.150562000 & 3.529027000 \\ 1 & 1.119835000 & 0.608360000 & 4.869769000 \\ 1 & -1.680906000 & 4.603281000 & 5.469496000 \\ 1 & 1.463431000 & 2.057003000 & 6.811640000 \\ 1 & 0.066015000 & 4.064964000 & 7.124774000 \\ 6 & 0.487718000 & 0.336591000 & 2.179329000 \\ 6 & 0.745526000 & -1.027480000 & 2.102830000 \\ 6 & 1.388971000 & 1.220133000 & 1.589248000 \\ 6 & 1.868916000 & -1.501404000 & 1.440506000 \\ 6 & 2.505202000 & 0.748993000 & 0.922328000 \\ 6 & 2.748860000 & -0.614859000 & 0.845867000 \\ 1 & 0.070606000 & -1.720859000 & 2.573105000 \\ 1 & 1.211032000 & 2.278711000 & 1.653155000 \\ 1 & 2.054443000 & -2.559743000 & 1.394508000 \\ 1 & 3.186138000 & 1.443923000 & 0.464299000 \\ 1 & 3.619613000 & -0.979907000 & 0.331288000\end{array}$

